\title{
TOWARDS RADIOMETRICAL ALIGNMENT OF 3D POINT CLOUDS
}

\author{
H. A. Lauterbach, D. Borrmann, A. Nüchter
}

Informatics VII - Robotics and Telematics, Julius-Maximilians University Würzburg, Germany

(helge.lauterbach, dorit.borrmann, andreas.nuechter) @uni-wuerzburg.de

\section{Commission II}

KEY WORDS: Laser scanning, Color correction, Colored Pointcloud, Radiometrical Alignment

\begin{abstract}
:
3D laser scanners are typically not able to collect color information. Therefore coloring is often done by projecting photos of an additional camera to the $3 \mathrm{D}$ scans. The capturing process is time consuming and therefore prone to changes in the environment. The appearance of the colored point cloud is mainly effected by changes of lighting conditions and corresponding camera settings. In case of panorama images these exposure variations are typically corrected by radiometrical aligning the input images to each other. In this paper we adopt existing methods for panorama optimization in order to correct the coloring of point clouds. Therefore corresponding pixels from overlapping images are selected by using geometrically closest points of the registered 3D scans and their neighboring pixels in the images. The dynamic range of images in raw format allows for correction of large exposure differences. Two experiments demonstrate the abilities of the approach.
\end{abstract}

\section{INTRODUCTION}

In the past years some devices have come to market that have an integrated camera, but the resulting color information are sometimes of limited quality. In order to create a consistent colored point cloud, some manufactures use high dynamic range techniques. However often 3D laser scanners are not able to collect color information. Therefore coloring is often done by projecting photos of an additional camera to the 3D scans. Commonly several pictures per scan are required to cover the laser scanners field-of-view. This capturing process is time consuming and therefore prone to changes in the environment. Besides dynamic objects in the scene the appearance of the colored pointcloud is mainly effected by changes of lighting conditions which correspond to variation in exposure and white balance of the captured images.

The RGB values of overlapping images are related to each other by the scene radiance. For short time intervals e.g. between two consecutive images, the scene radiance is assumed to be constant, so color changes are caused by the imaging system. The relation of radiance and its corresponding image point is usually modeled by a vignetting term considering the radial fall off of the lens and the camera response function, which models the non-linear processes within the camera. Both are determined either by precalibration or simultaneously during the alignment of the images.

In case of point clouds consisting of several colored 3D scans the assumption of constant scene radiance does not hold true since there may be a time gap of at least a couple of minutes up to several days between two consecutive scans. In absence of laboratory condition the environmental conditions do change. For instance Figure 1 depicts two images of a wall from different perspective. On the bottom the corresponding point cloud is shown, where the red line connects the scan positions. Obviously there is a large exposure difference between both images, which were captured on different days.

In this paper we radiometrically correct the point clouds by adapting existing methods for panorama optimization to point clouds. Corresponding pixels from overlapping images are selected by

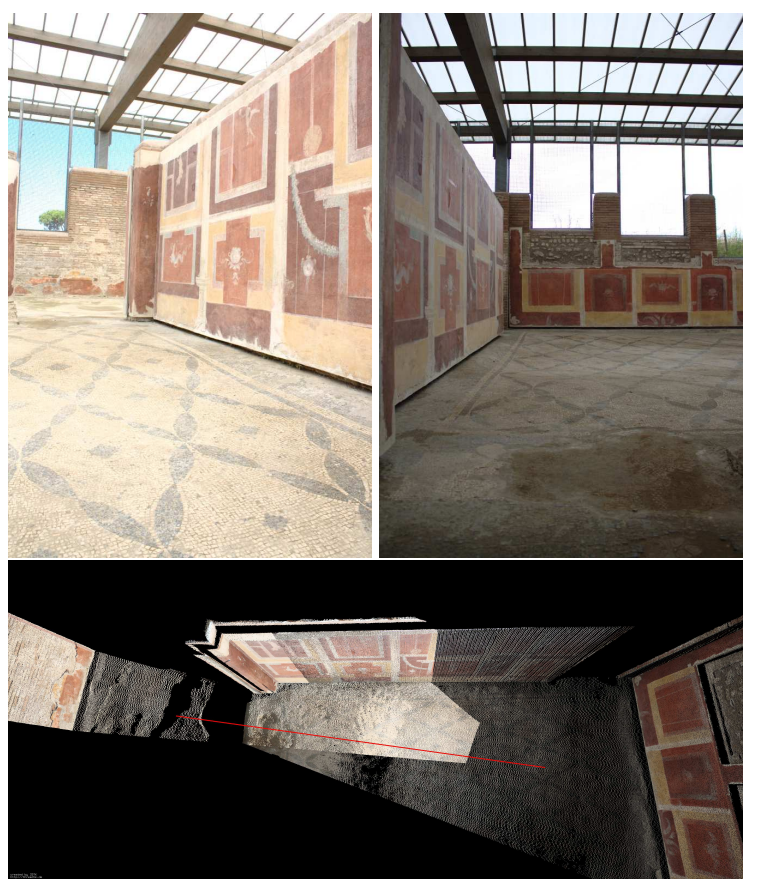

Figure 1. Point cloud of a wall (bottom). The images used for colorization (top) are not radiometrical aligned so the differences in exposure are clearly visible.

using geometrically closest points of the registered 3D scans. We also utilize the capabilities of most contemporary consumer cameras to store images in a raw format. This allows for subsequent correction of large exposure differences due to the higher dynamic range of the camera in comparison to 8bit RGB images. The remainder of this paper is organized as follows: Related work gives a survey of existing methods for radiometrical alignment of panorama images, while Image Formation recapitulates the image formation process. The following part summarizes our approach to correct for exposure differences in point clouds. Two experiments demonstrate the abilities of the approach. 


\section{RELATED WORK}

(Kanzok et al., 2012) locally correct colors during rendering a point cloud. Using the HSV color space they average the luminance channel of surface points which are projected to the same pixel, while hue and saturation are fixed. This provides local consistency in overlapping areas but does not produce globally consistent results. A change in hue and saturation is not considered, which may occur. In contrast (Agathos and Fisher, 2003) globally correct for color discontinuities in textured range images by computing a linear transformation matrix in RGB space which maps the color of one image to the other. In a second step they apply local corrections to smooth out small color variation boundaries.

(Eden et al., 2006) compute seamless high dynamic range panoramas by radiometrical aligning the input images. Therefore they compute white balance gains between radiance maps of overlapping images. They use a pre-calibrated camera to obtain the radiance map but they do not consider vignetting.

(d'Angelo, 2007) avoids to directly compute a radiance map. Instead from a set of corresponding point pairs the gray value transfer function is estimated, from which the corrected exposure value, the response function and the vignetting parameters are recovered.

A simple and fast method is to model the exposure differences between two images as well as the vignetting by a polynomial, thus avoiding to use the radiance space (Doutre and Nasiopoulos, 2009). By using only additive Terms the solution can be obtained by linear Regression. Further images are consecutively aligned to already corrected images. Therefore small errors in alignment sum up and a loop closure is not considered.

Color correction of point clouds is very similar to that of panorama images. However, large changes of perspective are typically not considered during panorama stitching. We radiometrical align images by using the 3D model to find geometrical corresponding pixels from overlapping source images, similar to (Agathos and Fisher, 2003). These correspondences are then radiometrically aligned exploiting the dynamic range of the raw images produced by the camera.

\section{IMAGE FORMATION}

In order to eliminate color artefacts such as seams and speckled patterns from overlapping images globally one strategy is to radiometrically align the images by finding a transform, which maps the color of one image to the color of the other image. Therefore knowledge about the relationship between a point in the scene and its corresponding image intensity or between two overlapping image points respectively, is required.

Figure 2 illustrates the image formation process in a simplified model. A point in the scene is lit by a light source and reflects the light towards the lens with radiance $L$, which is defined as the radiant power per solid unit angle and per unit projected area. $L$ is constant but depends on the angle under which the object point is seen, except for lambertian surfaces which reflect light diffuse.

The amount of light passing the lens and hitting the sensor with irradiance $E$ is mainly affected by the aperture $k$ and a radial falloff, known as vignetting. Due to the geometry of the lens the irradiance depends on the angle between the ray and the optical axis. In simple lenses this is often modeled as the $\cos ^{4}(\theta)$ law. $E$ is related to $L$ as follows:

$$
E=L \frac{\pi}{4}\left(\frac{1}{k}\right)^{2} \cos ^{4} \theta
$$

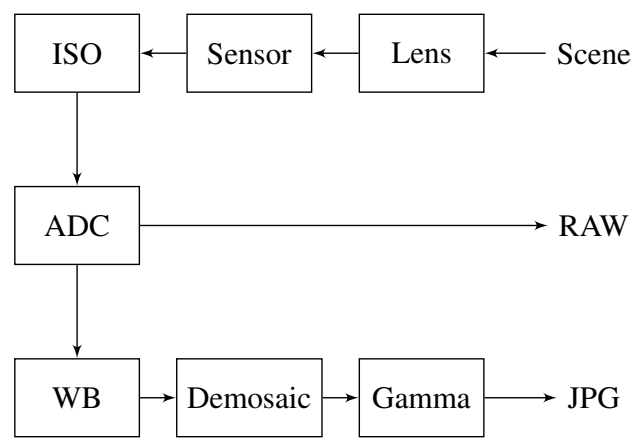

Figure 3. typical processing steps in the image formation.

A more general approach that also includes a falloff from other sources, is to model the spatial variation as a function $M(x)$ with respect to the image coordinates $x$ of a point, such that equation 1 generalizes to

$$
E=M(x) \frac{1}{k^{2}} L
$$

As proposed by (Goldman and Chen, 2005) $M$ is often modeled as a sixth degree even polynomial

$$
M(r)=1+\alpha r^{2}+\alpha_{2} r^{2}+\alpha_{3} r^{6}
$$

where $r$ is the distance with respect to the optical center.

The sensor integrates $E$ with shutter time $t$ which results in the exposure $H$.

$$
H=E t=e M L
$$

where $e=\frac{t}{k^{2}}$ represents the exposure settings The sensed signal is amplified and digitalized and either stored in a raw image format or further processed before storing in a jpeg image. In case of raw images, the images processing needs to be done afterwards.

As figure 3 depicts, important image processing steps are white balancing, demosaicking and compression of the dynamic range. White balancing reduces a hue from colored light by moving the white point of the image to pure white by balancing the color channels. Most often camera sensors use a color filter array in order to gather RGB information. Each pixel of the sensor provides only information for one channel, so the missing values have to be interpolated from neighboring pixels. Further image processing steps include a transform to a standardized color space, compression of the dynamic range. Camera often perform additional built-in image processing to boost the appearance of the images.

The aforementioned processing steps are camera specific and often nonlinear and not reversible. The relationship between the sensed exposure $E t$ and the final intensity $B$ in the image is modeled by a camera response function $f$.

$$
B=f(E t)=f(e M L)
$$

Most often $f$ is monotonous increasing and thus the inverse response function exists, which is used to compute the scene radiance. The relationship between two corresponding image intensities $B_{1}$ and $B_{2}$ regarding the radiance $L$ is described by the brightness transfer function $\tau$ (d'Angelo, 2007)

$$
B_{1}=\tau\left(B_{2}\right)=f\left(\frac{e_{1} M_{1}}{e_{2} M_{2}} f^{-1}\left(B_{2}\right)\right)
$$

Color images handled by either using a separate response function for each channel or by a single response function and additionally weighting the channels with a white balance factor $w$. 


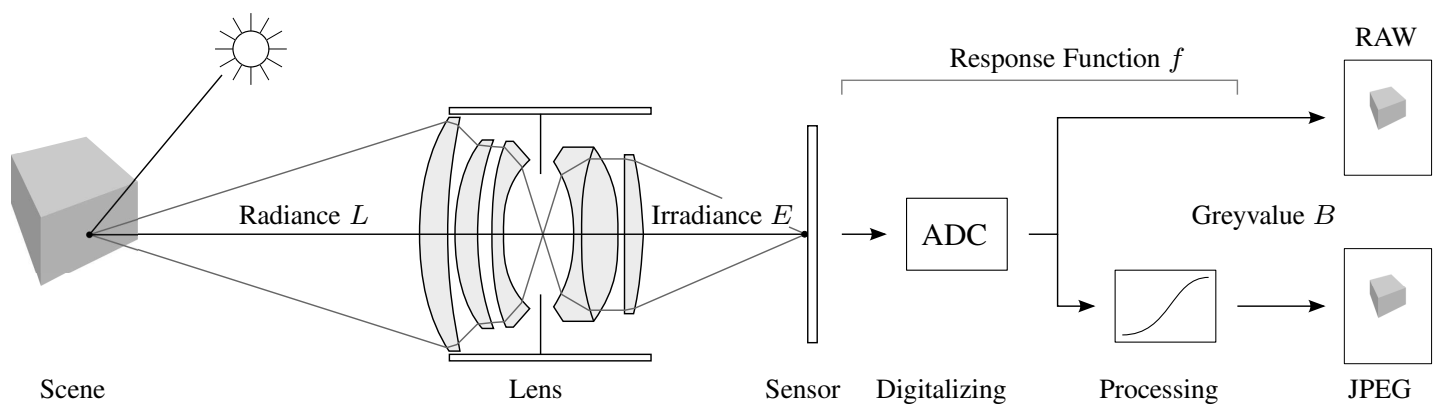

Figure 2. simplified model of radiometric image formation

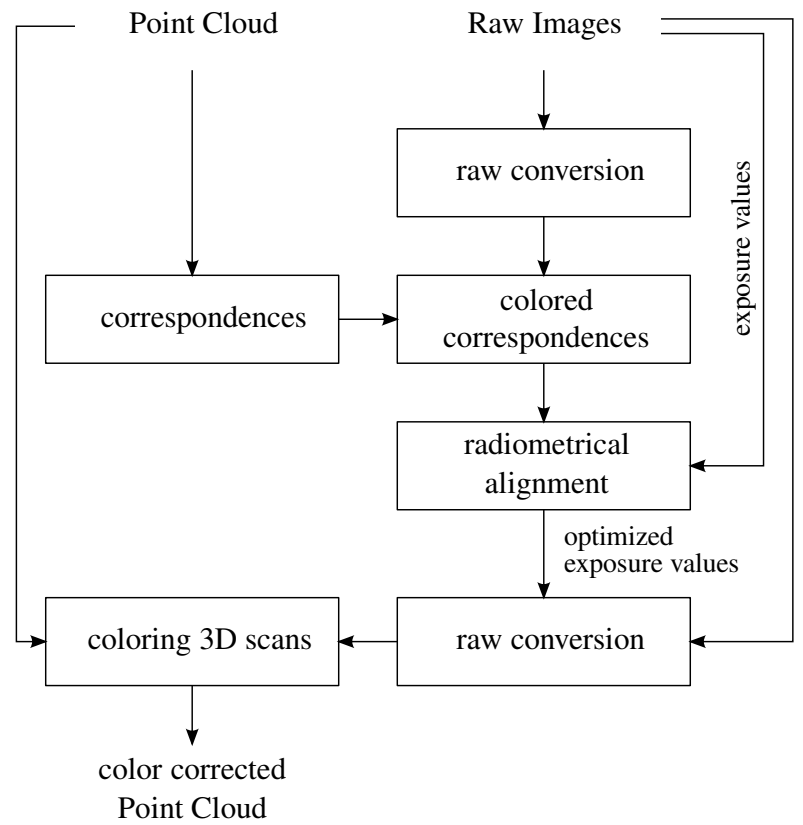

Figure 4. framework for radiometrical aligning point clouds

Equation 6 then becomes

$$
B_{1}=\tau\left(B_{2}\right)=f\left(\frac{w_{1} e_{1} M_{1}}{w_{2} e_{2} M_{2}} f^{-1}\left(B_{2}\right)\right)
$$

\section{RADIOMETRICAL ALIGNMENT OF POINT CLOUDS}

The problem of radiometrically aligning a point cloud consisting of several 3D scans is separable in two subproblems. The first problem, aligning the images of one single 3D scan is in fact similar to panorama stitching. A camera mounted on top of a laser scanner is only moved by rotating the scanner, so the translational movement is small. In this case the observed scene radiance for corresponding image points from overlapping images does not change in theory. The second problem is the alignment of images from overlapping 3D scans. Due to large changes of the perspective and the large time between capturing the images the observed radiance of a scene point is not constant for two corresponding image points. However, assuming scenes with predominantly lambertian surfaces we treat the observed radiance to be constant. As $L$ is proportional to $E$ we interpret differences as errors in the exposure settings like shutter time and white balance factors instead.

Figure 4 gives an overview of the processing steps of our ap- proach. We start by initially converting the raw images. Built in jpeg-conversion of the camera in general is not reproducable. Processing steps such as automatic exposure correction are included which are hard to model. Initially converting RAW images with minimal necessary steps keeps the impact of the non linear image processing at a minimum and ensures the response function to be the same in all steps of our approach. For conversion we use UFRaw (Fuchs, 2014) which allows to adjust the brightness of an image in aperture stops (EV) and batch processing. After that a set of corresponding point pairs is selected from the point cloud and colored with the previously converted images. This step is discussed in more detail in Section 5.

In the optimization step the images are radiometrical aligned. In this work we use the method of (d'Angelo, 2007). It minimizes the distance

$$
\varepsilon=d\left(b_{1}-\tau\left(B_{2}\right)\right)
$$

between an image intensity $B_{1}$ and the transfered corresponding intensity $B_{2}$ using the method of Levenberg-Marquart. By estimating the function $\tau$ the exposure values and white balance factors of the aligned images, as well as the camera response function are recovered.

Although the method of (d'Angelo, 2007) is able to estimate the vignetting simultaneously, this option was not considered. For estimating the vignetting the assumption of constant radiance is mandatory, which is not the case for more than one scan, due to large camera movements and time gaps. However, the vignetting can be integrated by pre calibration of the specific camera lens combination.

Differences in the white balance are considered by scaling the red and blue channel of each image with an additional white balance factor $w$ in $\tau$ in equation 6 . Note that this white balance factor differs from the cameras white balance. Demosaicking algorithms expect the raw image to be white balanced, so UFRaw first applies the white balancing. As we are working in RGB space the cameras white balance factors cannot be adjusted easily during the alignment process without applying the raw conversion for each image in each iteration. Instead we apply a second white balance which balances the interpolated RGB channels.

The initial guess for the exposure value of each image if derived from Exif data, which stores information about camera settings. One well exposed image is manually selected as reference image, to which the others are aligned to. The exposure value and white balance for this images is fixed during alignment.

Finally the computed optimized exposure values and white balance factors are used to generate the color corrected pointcloud from the converted camera raw images. 


\section{SELECTION OF CORRESPONDENCES}

In case of panorama images radiometrical corresponding pixels are usually found by first aligning the images with feature based registration and then sampling overlapping image regions.

From calibration of the 3D laser scanner and the camera the transformation between the local coordinate systems of both sensors is known (Borrmann et al., 2012). So each 3D point is assigned to one pixel of an image. Correspondences within one scan are determined by checking whether one 3D point is associated to more than one image.

Point pairs between images of overlapping scans are found by searching for geometrical closest points in the scans with a maximum distance below a threshold, e.g. $0.5 \mathrm{~mm}$. This is effectively done by using a kd-Tree representation of the scans.

However, the radiometric accuracy of the selected point pairs is not precise, as it is affected by

- the resolution of camera and laser scanner. Since the pixel density of camera images is usually higher than the point density of a laser scan, more than one candidate of image points per $3 \mathrm{D}$ points are available, thus there is a high probability of selecting the wrong one during coloring the scan.

- the accuracy of the calibration of laser scanner and camera. A displacement leads to selection of geometrical correct but radiometrical incorrect point pairs.

- the accuracy of scan registration, which has smaller but similar effects as the calibration

To reduce the impact of these factors the neighborhood of a point pair candidate is considered by taking the mean RGB value. By additionally calculating the gradient of an image point, point pairs with pixels lying on edges are rejected.

\section{EXPERIMENTS AND RESULTS}

The approach was tested in two experiments. In both scenarios the point clouds consist of several 3D scans acquired by a Riegl VZ-400 laser scanner. Additionally a Canon EOS 1000D DSLR camera is mounted on top of the scanner in order to colorize the laser scans. The calibration is done as described in (Borrmann et al., 2012). The camera is equipped with an EF-S 18-55 mm zoom lens, which is fixed at $18 \mathrm{~mm}$ focal length and manual focus.

In order to cover the horizontal field-of-view of the laser scanner of $360^{\circ}$ a minimum of 9 images per scan are needed, resulting in point cloud with 19 million colored $3 \mathrm{D}$ points and a vertical fieldof-view of $63^{\circ}$. The camera images provide more than 90 million images points, so approximately 5 candidate pixel per 3D point are available for coloring the scan.

The dataset for the first experiment was collected in Bremen, Germany at the excavation site of the rediscovered Stephanitorzwinger, which is depicted in Figure 5. This tower was part of the town fortification of Bremen in the 16th century. It was destroyed in the 17 th century, when a thunderstorm set the stored munitions to fire.

The dataset consists of eight terrestrial 3D scans and 10 images per scan, acquired with the above mentioned hardware configuration. The exposure settings were adjusted once and kept fixed

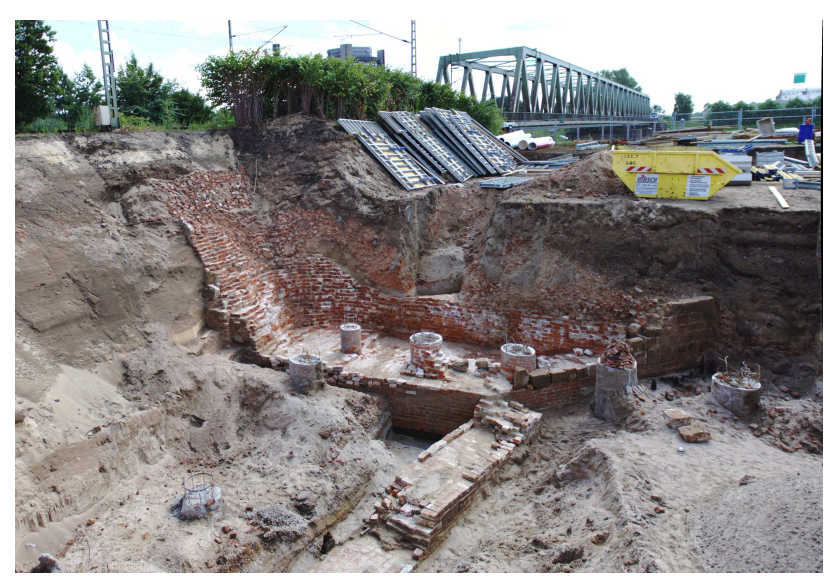

Figure 5. Excavation of the rediscovered Stephanitorzwinger in Bremen, Germany.

Table 1. Exposure value corrections applied to images for radiometrical alignment for the data set Stephanitorzwinger.

\begin{tabular}{l|rrrrrrrr} 
& \multicolumn{7}{|c}{ Scan } & \\
Img & 0 & 1 & 2 & 3 & 4 & 5 & 6 & 7 \\
\hline 0 & 1.96 & -0.25 & 0.13 & 0.00 & 0.05 & 1.13 & 0.97 & -0.19 \\
1 & 1.65 & 0.94 & -0.10 & -0.13 & 0.02 & 0.60 & 0.84 & -0.07 \\
2 & 1.64 & 0.88 & 0.32 & -0.02 & 0.10 & 0.11 & 0.35 & 0.02 \\
3 & 1.53 & 0.85 & 0.30 & -0.05 & 0.28 & -0.19 & -0.13 & 0.01 \\
4 & 1.39 & 0.60 & 0.02 & -0.14 & 0.08 & -0.07 & -0.22 & -0.18 \\
5 & 1.47 & 0.38 & -0.14 & 0.51 & -0.17 & 1.69 & 0.03 & -0.30 \\
6 & 1.23 & 0.66 & -0.27 & 0.64 & -0.20 & 1.79 & 0.09 & -0.13 \\
7 & 0.87 & 1.00 & -0.32 & 1.20 & -0.19 & 1.82 & 0.22 & -0.06 \\
8 & 1.03 & 1.24 & 0.22 & 1.31 & -0.08 & 1.96 & 0.09 & -0.23 \\
9 & 1.73 & 1.24 & 0.15 & 1.30 & 0.01 & 1.82 & 0.32 & -0.21
\end{tabular}

for all images. The measurements took several hours, so the exposure of the captured images was influenced by the position of the sun, but mainly by clouds obscuring the sun.

Figure 6 gives an example of the point cloud which illustrates the improvements of the radiometric optimization. The original point cloud is shown on the left. The scattered pattern on the depicted wall originates from overlapping images from different 3D scans.

Table 1 gives the resulting correction parameters $\Delta E V$ after optimization for each source image of the point cloud. While the environmental conditions are quite stable within scan 4 and 7 , they do change rapidly within others. Note the distance of more than 1.5 EV between consecutive images, e.g. images 4 and 5 in scan 5. For example the seam in Figure 6, marked with red arrows, is caused by the exposure distance of $0.69 \mathrm{EV}$ between the first and the last image of scan 5, which is not visible in the corrected point cloud anymore.

After exposure optimization the homogeneity of the texture increased, as shown on the right. The scattered pattern disappeared. Note that the remaining dark areas (marked with green arrows) originate from images, where these parts are in the shadows but the main part of the image are in bright sunlight. Therefore the images are even slightly darkened (Table 1 , image 0 and 1 of scan 7) and the parts in the shadows are not consistant with overlapping images.

The second dataset was recorded in one of the hadrianic garden houses in Ostia Antica, Italy, the ancient harbor city of Rome.(Ostia Antica, 2016) The hardware configuration as described above was mounted on the remote controlled mobile robot Irma3D (Borrmann et al., 2015). Measurements were distributed over five entire days, so the changes of environmental conditions are strong, partially even between consecutive images. The exposure set- 

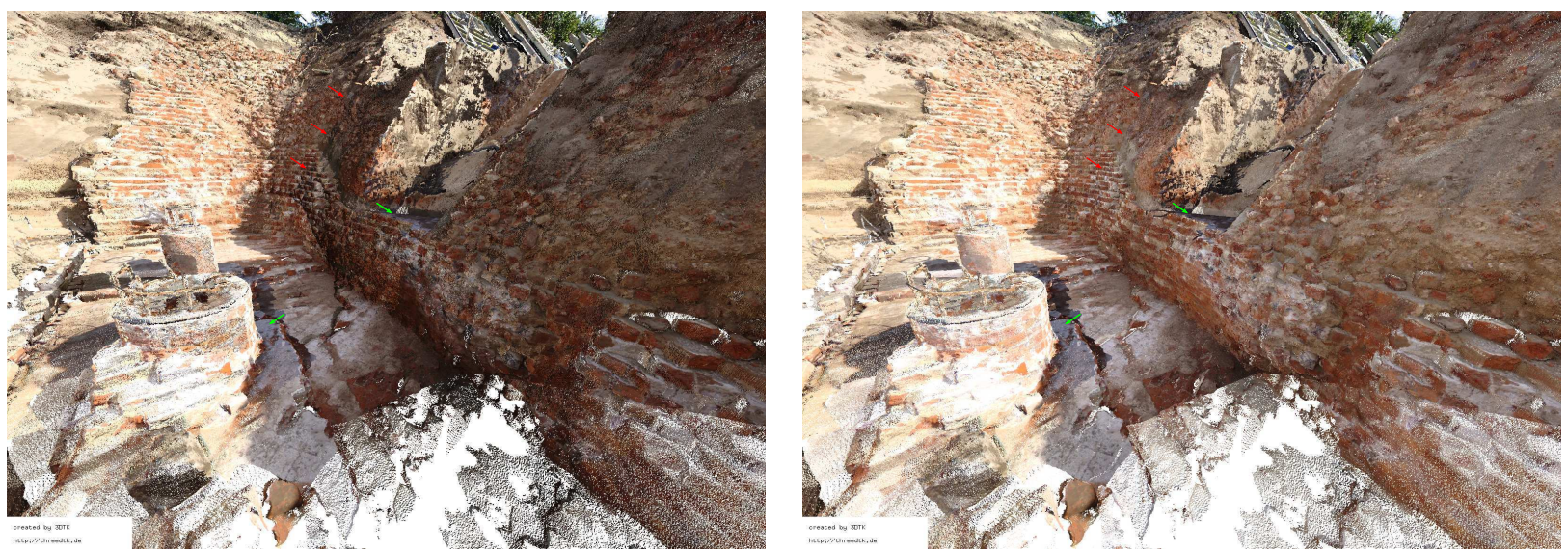

Figure 6. Stephanitorzwinger, Bremen, Germany. Details from the point cloud before (left) and after optimization (right) with equal point density. Note the scattered dark pattern on the brick wall, which disappeared after exposure correction.

tings were adjusted daily before starting the measurements and kept constant over the entire day.

The dataset consist of 56 scans in total, out of which 13 scans that cover the main room and the hallway of the garden house are used in this experiment. From the 117 source images in total 111999 corresponding point pairs are randomly selected for the exposure optimization.

The original uncorrected point cloud is depicted in Figure 7 on the left. Like in the first dataset changes in lighting conditions express themselves in the scattered pattern in regions of equal point density. Moreover hard seams appear due to image boundaries and occlusions. For instance this is visible behind the right pillars in the upper row and on both pillars in the bottom part of Figure 7.

Comparing the original point cloud in Figure 7 on the left to the optimized one on the right, the improvements are clearly visible. Despite the calibration inaccuracies, over- and underexposed images are adjusted, so the homogeneity in brightness distribution increases. Although the scattered pattern does not disappear completely, the texture of the floor becomes visible. Also the seams on the pillars (bottom row of Figure 7) reduce.

The paintings on the walls appear blurred both before and after exposure alignment. This is caused by the calibration between scanner and camera, which is not as precise as in the first experiment.

\section{CONCLUSIONS AND OUTLOOK}

In this paper radiometrical alignment of panorama images was adopted to point clouds. Both problems are similar to each other, but care has to be taken when selecting corresponding point pairs from a point cloud. In this work we used geometrical closest points and averaged over the neighborhood of an image point. Two experiments showed that this method improves the appearance of the point cloud but results depend on the registration between camera and laser scanner.

One idea to further reduce color difference is, to divide the radiometric optimization in two steps. At fist radiometrically align the images within one scan. Afterwards treat the aligned images of one scan as one single image and align the images of the scans. Further work will also concentrate on improving the quality of the corresponding point pairs by including feature based methods.

\section{REFERENCES}

Agathos, A. and Fisher, R. B., 2003. Colour texture fusion of multiple range images. In: 3-D Digital Imaging and Modeling, 2003. 3DIM 2003. Proceedings. Fourth International Conference on, IEEE, pp. 139-146.

Borrmann, D., Afzal, H., Elseberg, J. and Nüchter, A., 2012. Mutual calibration for $3 \mathrm{~d}$ thermal mapping. IFAC Proceedings Volumes 45(22), pp. 605-610.

Borrmann, D., Heß, R., Houshiar, H., Eck, D., Schilling, K. and Nüchter, A., 2015. Robotic mapping of cultural heritage sites. The International Archives of Photogrammetry, Remote Sensing and Spatial Information Sciences 40(5), pp. 9.

d'Angelo, P., 2007. Radiometric alignment and vignetting calibration. Proc. Camera Calibration Methods for Computer Vision Systems.

Doutre, C. and Nasiopoulos, P., 2009. Fast vignetting correction and color matching for panoramic image stitching. In: 2009 16th IEEE International Conference on Image Processing (ICIP), IEEE, pp. 709-712.

Eden, A., Uyttendaele, M. and Szeliski, R., 2006. Seamless image stitching of scenes with large motions and exposure differences. In: Computer Vision and Pattern Recognition, 2006 IEEE Computer Society Conference on, Vol. 2, IEEE, pp. 2498-2505.

Fuchs, U., 2014. Ufraw. Webpage. http://ufraw.sourceforge.net/.

Goldman, D. B. and Chen, J.-H., 2005. Vignette and exposure calibration and compensation. In: Computer Vision, 2005. ICCV 2005. Tenth IEEE International Conference on, Vol. 1, IEEE, pp. 899-906.

Kanzok, T., Linsen, L. and Rosenthal, P., 2012. On-the-fly luminance correction for rendering of inconsistently lit point clouds.

Ostia Antica, 2016. SOPRINTENDENZA SPECIALE PER I BENI ARCHEOLOGICI DI ROMA - SEDE DI OSTIA. Webpage. http://www.ostiaantica.beniculturali.it. 
The International Archives of the Photogrammetry, Remote Sensing and Spatial Information Sciences, Volume XLII-2/W3, 2017 3D Virtual Reconstruction and Visualization of Complex Architectures, 1-3 March 2017, Nafplio, Greece

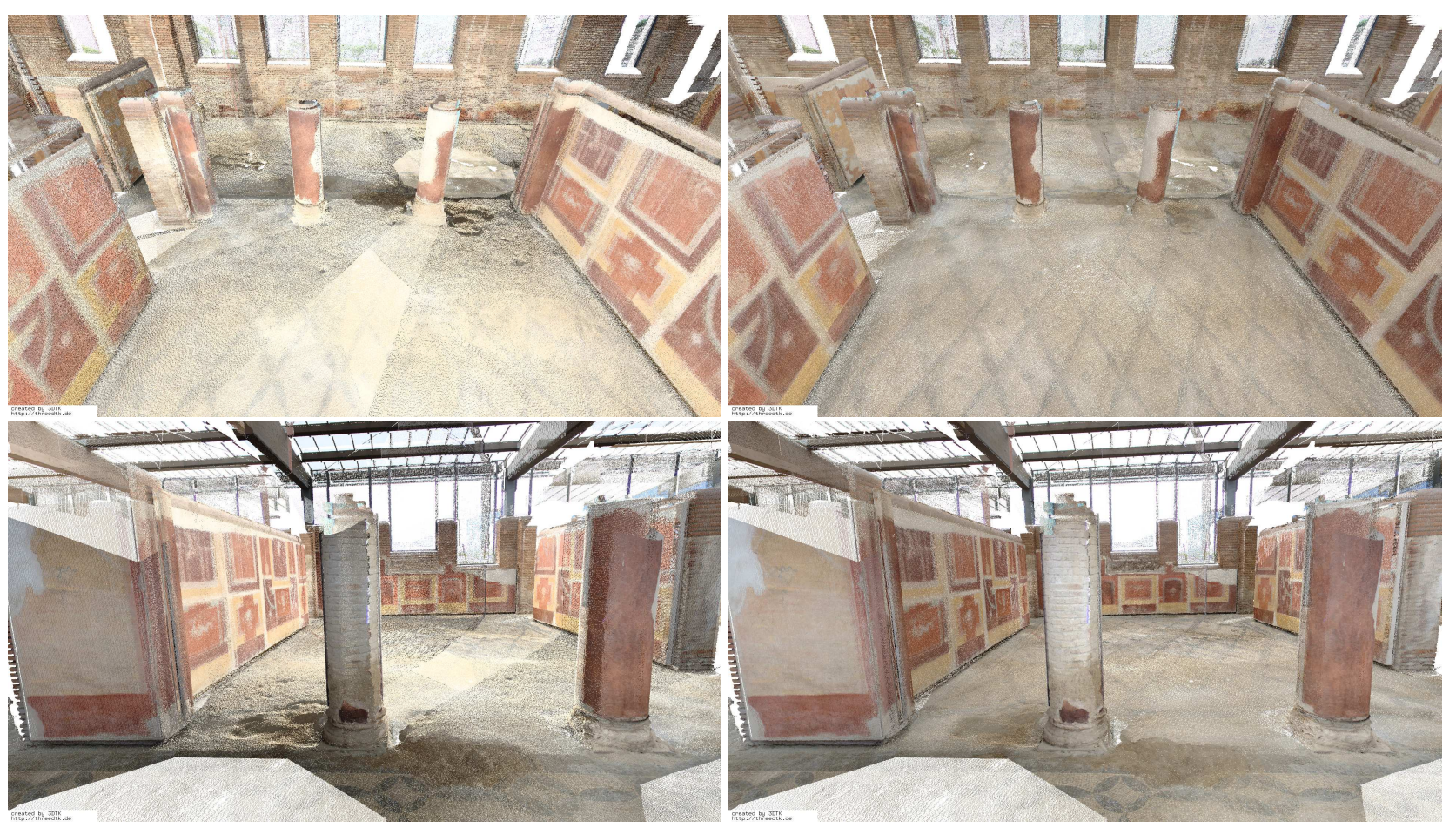

Figure 7. Ostia Antica. Details from point cloud before (left) and after optimization (right). Over- and underexposed images are aligned, therefore the homogeneity in brightness distribution increased. The point density is exactly the same for all images. 